\title{
Efeito de diferentes intervalos entre as tentativas do teste de 1-RM no desempenho de força máxima em mulheres
}

\author{
Effect of different intervals between the attempts of the 1-RM test \\ performance in maximum strength in women
}

\author{
R.C. Paixão, M. Costa Junior, L.D. Galdino, G.G. Agostini, J.D. Nunes
}

ARTIGO ORIGINAL | ORIGINAL ARTICLE

\begin{abstract}
RESUMO
O teste de uma repetição máxima (1-RM) é frequentemente aplicado para medir a força de um indivíduo em um determinado exercício. O objetivo do presente estudo foi comparar o efeito de diferentes intervalos entre as tentativas do teste de 1-RM no desempenho de força máxima em mulheres no exercício leg press $45^{\circ}$. Para tanto, 12 voluntárias (19 \pm 1 ano) praticantes de exercícios resistidos há no mínimo seis meses foram submetidas a quatro sessões de testes, alternadas por no mínimo 48 horas de repouso. Os intervalos de recuperação entre as tentativas do teste de 1-RM foram de 30, 45, 60 e 180 segundos, sendo que a ordem de execução foi definida aleatoriamente. Para identificação da diferença entre os intervalos propostos foi aplicada a análise de variância ANOVA One-way $(p<.05)$. Os resultados não revelaram diferenças significativas no desempenho de força máxima em mulheres, quando comparados os valores médios de teste obtidos em cada um dos intervalos propostos. Esses achados indicam que para a medida de força máxima no exercício leg press $45^{\circ}$, intervalos menores do que um minuto (30 - 45 segundos) podem ser utilizados com a mesma precisão que intervalos maiores, garantindo maior economia de tempo para a aplicação do teste de 1-RM.

Palavras-chave: exercício, teste de 1-rm, recuperação, desempenho
\end{abstract}

ABSTRACT

Test of one repetition maximum (1-RM) is often used to measure the strength of an individual in a given exercise. The purpose of this study was to compare the effect of different intervals between the attempts of the 1-RM test on the maximum strength performance in women on the leg press $45^{\circ}$. Thus, 12 volunteers (19 \pm 1 year) resistance exercises to practice at least six months, were submitted to four sessions of testing, alternated by at least 48 hours. The recovery intervals between the attempts of the 1-RM test were 30, 45, 60 and 180 seconds, with order randomly determined. To identify the difference between the intervals proposed was applied to analysis of variance One-way ANOVA $(p<.05)$. The results revealed no significant differences in performance for maximal strength in women, compared to the average values obtained in each test interval. These findings indicate that for maximal strength measure in leg press $45^{\circ}$, intervals less than a minute (30 - 45 seconds) can be used with the same accuracy as longer intervals, providing greater time economy for 1-RM test application.

Keywords: exercise, 1-rm test, recovery, performance

Submetido: 29.04.2012 | Aceite: 15.05.2013

Rodney Coelho da Paixão, Marcelo Costa Junior, Leandro Cézar Domingos Galdino, Guilherme Gularte de Agostini, João Elias Dias Nunes. Universidade Federal de Uberlândia, Brasil.

Endereço para correspondência: João Elias Dias Nunes, Faculdade de Educação Física e Fisioterapia (FAEFI - UFU), Rua Benjamin Constant, 1286, CEP 38400-678, Bairro Aparecida, Uberlândia, Minas Gerais, Brasil.

E-mail: dneyudi@hotmail.com 
O aumento da força muscular tem se apresentado como um dos principais benefícios do treinamento resistido (TR), visto que essa qualidade física é decisiva para melhorar o desempenho atlético bem como a execução de atividades da vida diária (Kraemer \& Ratamess, 2004). Por sua vez, o TR pode ser prescrito em função da combinação de diversas variáveis, quais como a carga de treinamento e o intervalo de descanso entre as séries (American College os Sports Medicine [ACSM], 2009).

Nesse contexto, o teste de uma repetição máxima (1-RM), capaz de definir a maior carga que o indivíduo consegue levantar de maneira correta e em uma única vez (Slade, Miszko, Laity, Agrawal, \& Cress, 2002), é a avaliação mais comumente aplicada para a mensuração da força muscular dinâmica máxima (Brown \& Weir, 2001), servindo como um importante parâmetro de escolha para a percentagem de carga a ser utilizada durante os treinamentos, conforme os objetivos de cada sujeito.

Entretanto, embora tal teste seja considerado padrão-ouro para a mensuração da força máxima em situações não-laboratoriais (Levinger et al., 2007), é comum encontrar na literatura protocolos bastante diferentes em relação à sua aplicabilidade, principalmente no que diz respeito ao intervalo entre as tentativas (Miszko et al., 2003; Tracy, Byrnes, \& Enoka, 2004; Schroeder et al., 2007; DiFrancisco-Donoghue, Werner, \& Douris, 2007).

Miszko et al. (2003) ao verificarem o efeito de um treinamento de força e potência em homens e mulheres com idade média de $72.5 \pm$ 6.3 anos, adotaram um protocolo para avaliação da força muscular máxima, onde o intervalo entre as tentativas foi igual ou superior a três minutos. Em contrapartida, DiFrancisco-Donoghue et al. (2007) ao investigarem o efeito de duas diferentes freqüências de treinamento resistido em uma população idosa, aplicaram o teste de 1-RM para seis exercícios diferentes, utilizando para isso intervalos de apenas 30 segundos entre as tentativas em um mesmo aparelho e dois minutos de intervalo entre um aparelho e outro.

Assim, observamos que embora existam grandes diferenças em relação ao tempo de descanso adotado entre as tentativas dos testes de 1-RM, poucas pesquisas se propuseram a comparar o efeito que a manipulação dessa variável pode causar sobre os resultados finais de força obtidos neste tipo de avaliação (Matuszak, Fry, Weiss, Ireland, \& McKnight, 2003; Weir, Wagner, \& Housh, 1994). Além disso, os raros trabalhos encontrados na literatura investigaram somente intervalos iguais ou superiores a um minuto e contaram com uma população amostral exclusivamente do gênero masculino, que por vez apresenta características físicas e metabólicas diferentes em relação ao gênero feminino (Burd, Tang, Moore, \& Phillips, 2008), podendo este ser um fator influente sobre o rendimento do teste.

Portanto, o objetivo deste estudo foi comparar o desempenho de força máxima em mulheres aparentemente saudáveis, quando submetidas a intervalos de 30, 45, 60 e 180 segundos entre as tentativas do teste de 1-RM no exercício leg press $45^{\circ}$. Nossa hipótese de trabalho foi de que mesmo em situações de teste envolvendo intervalos mais curtos o desempenho final não seria comprometido, sendo o número limitado de tentativas um dos fatores determinantes para isso.

\section{MÉTODO}

\section{Amostra}

Doze mulheres $(19 \pm 1$ ano; $57.9 \pm 4.64$ $\mathrm{kg} ; 165.58 \pm 4.66 \mathrm{~cm}$ ), aparentemente saudáveis participaram voluntariamente deste estudo. Como critérios de inclusão as participantes deveriam praticar exercícios resistidos regularmente ( $>$ duas vezes por semana) há no mínimo seis meses, não portarem quaisquer enfermidades osteomioarticulares que pudessem influenciar a execução correta do exercício e não realizarem suplementação de creatina. Todas as voluntárias assinaram um 
termo de consentimento livre e esclarecido, e foram informadas dos riscos e benefícios envolvidos nos procedimentos adotados. A massa corporal dos sujeitos foi mensurada em uma balança digital (Micheletti, Brasil) e a estatura foi obtida em um estadiômetro (Sanny, Brasil).

O protocolo experimental seguiu a resolução 196/96 do Conselho Nacional de Saúde, sendo aprovado pelo Comitê de Ética e Pesquisa da Universidade Federal de Uberlândia sob o protocolo CEP/UFU 393/10.

\section{Instrumentos}

O exercício leg press $45^{\circ}$ (GN Sport, Brasil) foi escolhido por ser bastante popular no TR de indivíduos com diferentes níveis de treinamento. Esse fato pode ser parcialmente explicado por esse exercício ser multiarticular, envolver grandes grupos musculares e possuir muitas similaridades mecânicas e neuromusculares com movimentos atléticos (Escamilla et al., 2001).

\section{Procedimentos}

Para a realização desse estudo, as voluntárias compareceram ao Laboratório de Fisiologia do Desempenho (LAFIDE), da Universidade Federal de Uberlândia, em quatro dias alternados por, no mínimo, 48 horas.

Os intervalos de recuperação entre as tentativas do teste de 1-RM foram de 30, 45, 60 e 180 segundos. A ordem de execução dos testes com diferentes intervalos foi definida aleatoriamente por meio de sorteio.

\section{Protocolo do Teste de 1-RM}

$\mathrm{O}$ protocolo adotado para o teste seguiu as recomendações de Brown e Weir (2001). O aquecimento consistiu de três séries. A $1^{\circ}$ série foi realizada apenas com o peso da plataforma do aparelho $(30 \mathrm{~kg})$ executando-se 20 repetições, a $2^{\circ}$ série com oito repetições e aproximadamente $50 \%$ da carga estimada de $1-\mathrm{RM}$, e a $3^{\circ}$ série com três repetições e aproximadamente $75 \%$ da carga estimada de 1-RM. Entre cada uma dessas séries foi concedido ao volun- tário um minuto de descanso. Ao fim da última série de aquecimento foram respeitados três minutos de intervalo antes da $1^{\circ}$ tentativa do teste propriamente dito. Foram concedidas no máximo cinco tentativas para obtenção da carga máxima levantada. A média de tentativas em cada sessão de testes, na corrente pesquisa, foi de $4.75 \pm 0.73$.

Antes do $1^{\circ}$ teste foi determinado, por meio de um goniômetro manual (Carci, Brasil), o ponto até o qual o voluntário deveria realizar a fase excêntrica do movimento $\left(90^{\circ}\right.$ de flexão do joelho). Dessa maneira, em todas as sessões o movimento iniciava pela fase excêntrica (1 - 2 segundos), e era concluído ao fim da fase concêntrica.

Ao longo de todos os testes, dois avaliadores proviam as condições de segurança e garantiam a execução correta do exercício. Conforme sugerido por McNair, Depledge, Brettkelly, \& Stanley (1996), foram utilizados incentivos verbais padronizados e realizados pelo mesmo avaliador.

\section{Análise Estatística}

Para a verificação de normalidade foi aplicado o Shapiro Wilk's test em todos os conjuntos de dados. Na sequiência, foi aplicada a análise de variância ANOVA One-way para identificação da diferença entre os intervalos propostos. $\mathrm{O}$ nível de significância adotado foi de $p<.05$. O software utilizado foi Statistica versão 7.0 (USA).

\section{RESULTADOS}

A Tabela 1 e a Figura 1 apresentam os resultados dos testes de 1-RM para os intervalos de 30, 45, 60 e 180 segundos. A análise

Tabela 1

Valores de 1-RM obtidos para cada um dos intervalos propostos (média \pm desvio padrão)

\begin{tabular}{cc}
\hline Intervalo $(\mathrm{s})$ & $1-\mathrm{RM}(\mathrm{kg})$ \\
\hline 30 & $236.6 \pm 48.3$ \\
45 & $253.3 \pm 58.6$ \\
60 & $240.0 \pm 60.3$ \\
180 & $245.8 \pm 47.1$ \\
\hline
\end{tabular}




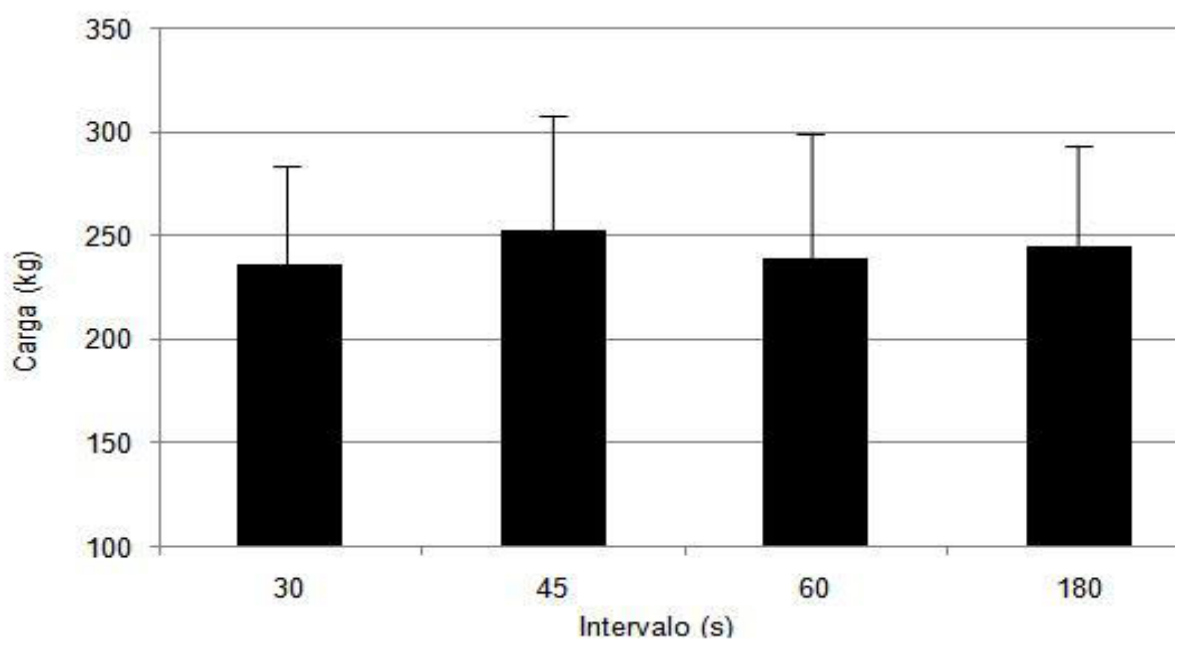

Figura 1. Desempenho de força máxima no teste de 1-RM com intervalos de 30, 45, 60 e 180 segundos entre as tentativas.

de variância ANOVA One-way, não identificou diferenças estatisticamente significativas entre os intervalos analisados $(p=.87)$.

\section{DISCUSSÃO}

O objetivo deste estudo foi comparar o desempenho de força máxima de mulheres aparentemente saudáveis quando submetidas a diferentes intervalos de recuperação entre as tentativas do teste de 1-RM. Nesse sentido, os resultados mostraram que não houve diferenças estatisticamente significativas no rendimento do teste de 1-RM para intervalos de 30, 45, 60 e 180 segundos (Tabela 1; Figura 1).

Esses achados vão ao encontro daqueles observados na pesquisa de Matuszak et al. (2003). Os autores analisaram o efeito de intervalos de um, três e cinco minutos sobre o rendimento de força máxima em homens com experiência em $\mathrm{TR}$, concluindo que um minuto de descanso entre as tentativas do teste de 1-RM foi suficiente para a recuperação do avaliado.

Nossos resultados corroboram também com os achados de Weir et al. (1994), que observaram o efeito de quatro intervalos distintos, sendo um, três, cinco e 10 minutos. De maneira semelhante à pesquisa supracitada, a amostra do estudo de Weir et al. (1994) também foi composta por homens habituados ao TR e os resultados não apontaram diferenças significativas entre os intervalos propostos.
Vale ressaltar, que o exercício analisado em nosso estudo (leg press $45^{\circ}$ ) para a determinação da carga de 1-RM, é diferente daqueles utilizados por Matuszak et al. (2003) e Weir et al. (1994), que aplicaram tal teste no agachamento e no supino reto, respectivamente. Nesse contexto, as evidências indicam que independentemente do exercício analisado envolver membros superiores ou inferiores, intervalos de repouso mais longos (5 - 10 minutos) não contribuem para um rendimento significativamente melhor no desempenho de força máxima. O mesmo não poderia ser afirmado até então a respeito de intervalos menores do que um minuto, devido à inexistência de trabalhos que fizeram essa análise. Logo, em nosso conhecimento, o presente estudo é o primeiro a comparar intervalos menores do que um minuto (30 e 45 segundos) em testes de 1-RM no exercício leg press $45^{\circ}$ e o seu efeito sobre o desempenho da força muscular máxima de membros inferiores. Ademais, sugere-se que intervalos mais curtos ( $<1$ minuto) podem ser utilizados na medida da força com precisão equivalente a intervalos mais extensos.

Adicionalmente, conforme já mencionado a corrente pesquisa diferencia-se daquelas conduzidas por Matuszak et al. (2003) e Weir et al. (1994), pois sua amostra foi composta exclusivamente por mulheres, que por sua vez apresentam características físicas e metabólicas dife- 
rentes dos homens (Burd et al., 2008). Dias et al. (2005) acompanharam durante oito semanas 38 indivíduos, de ambos os gêneros, que realizaram 10 exercícios envolvendo membros inferiores, tronco e membros superiores. Esses autores demonstraram que as mulheres apresentam um incremento relativo de força superior aos homens, o que garante a elas maior potencial para desenvolvimento da força muscular do que eles após curtos períodos de TR.

Embora cada sessão de testes tenha sido executada com um intervalo diferente entre as tentativas, em nenhuma delas foi realizada recuperação ativa da musculatura envolvida. Supõe-se que esse tipo de descanso facilitou o processo de ressíntese da fosfocreatina $(\mathrm{PCr})$, através de uma maior disponibilidade de oxigênio (Haseler, Hogan, \& Richardson, 1999), impedindo que houvesse uma queda brusca dos níveis de concentração muscular desse fosfato altamente energético mesmo quando utilizado tempos de intervalos menores (30 - 45 segundos).

Ademais, o protocolo empregado no presente estudo utilizou um número máximo de cinco tentativas para determinação da carga máxima, desta maneira, podemos sugerir que mesmo que o indivíduo fosse submetido a todas as tentativas possíveis, dificilmente os estoques de PCr seriam depletados a ponto de prejudicarem a execução do exercício e o rendimento final de cada voluntário (Gomes \& Aoki, 2005), independentemente do intervalo de repouso empregado ser mais curto ou mais longo.

Consideremos também que em atividades concêntricas máximas a fadiga periférica é desenvolvida primeiramente, seguida pela fadiga central (Babault, Desbrosses, Fabre, Michaut, \& Pousson, 2006). Como não houve diferenças no desempenho de força máxima nos intervalos de $30,45,60$ e 180 segundos, podemos inferir que tempos mais curtos de recuperação não favorecem o estabelecimento de fadiga periférica e consequentemente de fadiga central.

O estudo limitou-se à análise do efeito de diferentes intervalos entre as tentativas do teste de 1-RM por meio da comparação entre as cargas alcançadas. Para estudos futuros sugere-se a realização de procedimentos que busquem elucidar a relação e a exata importância das diversas fontes energéticas no suprimento das ações desenvolvidas durante o teste.

\section{CONCLUSÕES}

A partir dos resultados encontrados no presente estudo, concluímos que os intervalos de 30, 45, 60 e 180 segundos não apresentam diferenças estatisticamente significativas no desempenho de força máxima no exercício leg press $45^{\circ}$ em mulheres. Do ponto de vista prático, esses achados garantem maior economia de tempo para a aplicação do teste de 1-RM sem que a sua validade seja comprometida.

Agradecimentos:

Nada declarado.

Conflito de Interesses:

Nada declarado.

Financiamento:

Nada declarado.

\section{REFERÊNCIAS}

American College of Sports Medicine. (2009). Position stand on progression models in resistance training for healthy adults. Medicine $\mathcal{E}$ Science in Sports \& Exercise, 41 (3), 687-708. doi: 10.1249

Babault, N., Desbrosses, K., Fabre, M., Michaut, A., \& Pousson, M. (2006). Neuromuscular fatigue development during maximal concentric and isometric knee extensions. Journal of Applied Physiology, 100(3), 780-785. doi: 10.1152

Brown, L.E., \& Weir, J.P. (2001). ASEP Procedures recommendation I: accurate assessment of muscular strength and power. Journal of Exercise Physiology Online, 4(3), 1-21.

Burd, N.A., Tang, J.E., Moore, D.R., \& Phillips, S.M. (2009). Exercise training and protein metabolism: influences of contraction, protein intake, 
and sex-based differences. Journal of Applied Physiology, 106(5), 1692-1701. doi: 10.1152

Dias, R.M.R., Cyrino, E.S., Salvador, E.P., Nakamura, F.Y., Pina, F.L.C., \& Oliveira, A. R. (2005). Impacto de oito semanas de treinamento com pesos sobre a força muscular de homens e mulheres. Revista Brasileira de Medicina do Esporte, 11(4), 224-228. doi: 10.1590

DiFrancisco-Donoghue, J., Werner, W., \& Douris, P.C. (2007). Comparison of once-weekly and twice-weekly strength training in older adults. British Journal of Sports Medicine, 41(1), 19-22. doi: 10.1136

Escamilla, J.R., Fleisig, G.S., Zheng, N., Lander, J.E., Barrentine, S.W., Andrews, J.R., ... Moormam, C.T. (2001). Effect of technique variations on knee biomechanics during the squat and leg-press. Medicine \& Science in Sports \& Exercise, 33(9), 1552-1566. doi: 10.1249

Gomes, R.V., \& Aoki, M.S. (2005). Suplementação de creatina anula o efeito adverso do exercício de endurance sobre o subsequente desempenho de força Revista Brasileira de Medicina do Esporte, 11(2), 131-134. doi: 10.1590

Haseler, L.J., Hogan, M.C., \& Richardson, R.C. (1999). Skeletal muscle phosphocreatine recovery in exercise-trained humans is dependent on $\mathrm{O} 2$ availability. Journal of Applied Physiology, 86(6), 2013-2018. doi: 10.1152

Kraemer, W.J., \& Ratamess, N.A. (2004). Fundamentals of resistance training: progression and exercise prescription. Medicine $\mathcal{E}$ Science in Sports $\mathcal{E}$ Exercise, 36(4), 674-688. doi: 10.1249

Levinger, I., Goodman, C., Hare, D.L., Jerums, G., Toia, D., \& Selig, S. (2009). The reliability of the 1 RM strength test for untrained middle-aged individuals. Journal of Science and Medicine in Sport, 12(2), 310-316. doi: 10.1016
Matuszak, M.E., Fry, A.C., Weiss, L.W., Ireland, T.R., \& McKnight, M.M. (2003). Effect of Rest Interval Length on Repeated 1 Repetition Maximum Back Squats. Journal of Strength \& Conditioning Research, 17(4), 634-637. doi: 10.1519

McNair, P.J., Depledge, J., Brettkelly, M., \& Stanley, S.N. Verbal encouragement: effects on maximum effort voluntary muscle action. British Journal of Sports Medicine, 30(3), 243-245. doi: 10.1136

Miszko, T.A., Cress, M.E., Slade, J.M., Covey, C.J., Agrawal, S.K., \& Doerr, C.E. (2003). Effect of Strength and Power Training on Physical Function in Community-Dwelling Older Adults. Journal of Gerontology, 58(2), 171-175. doi: 10.1093

Schroeder, E.T., Wang, Y., Castaneda-Sceppa, C., Cloutier, G., Vallejo, A.F., Kawakubo, M., ... Sattler, F.R. (2007). Reliability of Maximal Voluntary Muscle Strength and Power Testing in Older Men. Journal of Gerontology, 62(5), 543-549. doi: 10.1093

Slade, J.M., Miszko, T.A., Laity, J.H., Agrawal, S.K., \& Cress, M.E. (2002). Anaerobic power and physical function in strength-trained and non-strength-trained older adults. Journal of Gerontology, 57(3), 168-172. doi: 10.1093

Tracy, B.L., Byrnes, W.C., \& Enoka, R.M. (2004). Strength training reduces force fluctuations during anisometric contractions of the quadriceps femoris muscles in old adults. Journal of Applied Physiology, 96(4), 1530-1540. doi: 10.1152

Weir, J.P., Wagner, L.L., \& Housh, T.J. (1994). The Effect of Rest Interval Length on Repeated Maximal Bench Presses. Journal of Strength \& Conditioning Research, 8(1), 58-60. doi: 10.1519 especificado em contrário e nos conteúdos retirados de outras fontes bibliográficas. 